\title{
Reconocimiento a revisores y dictaminadores
}

C on el presente volumen, Salud Pública de México cumple 40 años de existencia ininterrumpida, tiempo durante el cual ha alcanzado importantes logros como publicación científica, gracias, en gran parte, a la participación de diversos revisores. Por lo tanto queremos hacer patente nuestro reconocimiento a todos aquellos especialistas y colaboradores que desinteresadamente aportaron su valioso tiempo y conocimiento para evaluar y dictaminar la validez académica, relevancia y pertinencia de los artículos que recibimos durante los últimos doce meses.

En gran medida, las recomendaciones del revisor se constituyen en un verdadero faro de orientación, consejo y dirección para los investigadores. Esta tarea es más sorprendente si se toma en cuenta que el dictaminador, además de no percibir remuneración o crédito alguno por su trabajo, ni siquiera sabe quién es el beneficiario del apoyo y guía que brinda al efectuar un análisis concienzudo de los materiales que se le encomiendan. Este proceso se perfecciona con la receptividad de los autores que incorporan sugerencias e indicaciones, complementan o corrigen sin conocer tampoco de quién provienen, partícipes tan sólo de la mística que une a esta comunidad sabedora de la buena fe y seriedad de los académicos involucrados.

Además de sui generis, la tarea es enorme. Las tasas de rechazo, el número de segundas y terceras versiones y las revisiones que por la complejidad, novedad, o carácter polémico de un trabajo requieren dos, tres y cuatro dictaminadores acrecientan la hora/hombre, de alta calificación, dedicadas a esta labor. Es precisamente esta tarea de alto contenido académico la que debe ser considerada y tomada en cuenta por las comisiones calificadoras para el otorgamiento de estímulos y reclasificaciones del sistema de investigación en México. Es esencial que no transcurra más tiempo sin que esta actividad altamente especializada sea reconocida en su justa dimensión.

El número de revisores que cuentan con el prestigio intelectual y moral requerido y que están dispuestos a comprometer y sostener una empresa editorial que se torna demandante, no es muy extenso. Queremos agradecer especialmente a nuestros editores de sección, los doctores Mauricio Hernández, Mario Henry Rodríguez, Malaquías López, José Luis Valdespino y Miguel Angel Lezana. También, nos sentimos muy afortunados de haber contado este año con el apoyo de los siguientes especialistas.

Luis Martín Abreu Guadalupe Aguilar Madrid Héctor Gerardo Aguirre Gas Arnulfo Albores Medina Carlos Humberto Alvarez Lucas Hugo Amigo Hugo Hernando Aréchiga Urtuzuástegui Sofía Arjonilla Alday Armando Arredondo Abelardo Avila Curiel Armando Barriguete Castellón Federico Bonilla Marin Guilherme Luiz Guimaraes Borges
Víctor Hugo Borja Aburto

Viviane Brachet Staehling Humberto Bravo Alvarez Lilia Patricia Bustamante Montes Ernesto Calderón Jaimes Carlos Eduardo Carrillo Ordaz Susana Cerón Mireles Ignacio Chávez Rivera Adolfo Chávez Villasana José Cifuentes Enrique Cifuentes García Patricia Clarck Peralta Carlos J. Conde González
Andrew Collins

Alexánder Enrique Corcho Berdugo

Ma. del Carmen Cravioto

Patricia Cravioto Quintana

Carlos Cruz Rivero

Carlos del Río Chiriboga

Fernando Díaz Barriga

Juan Luis Gerardo Durán Arenas

Lilia Irene Durán González

Guy Duval Berhmann

Gabriela Echániz Aviles

Jorge Escobedo de la Peña

Josep A. Espinas 
Guillermo Fanghänel Salmón

Paulina Farías Serra

Francisco Fernández Pellón

Mario Efraín Flores Aldana

Javier Flores Murrieta

Teresa Fortoul

Silvestre Frenk Freund

Julio Frenk Mora

Howard Frumkin

María de Lourdes García García

Mariano García Viveros

Francisco Garrido Latorre

Nadine Gasman Zylbermann

Octavio Gómez Dantés

Héctor Gómez Dantés

Ruth González Serratos

Teresa González de Cossío

Clicerio González Villalpando

HéctorJoséFernando GuiscafréGallardo

Jesús Héctor Gutiérrez Avila

Luis Miguel Gutiérrez Robledo

Gonzalo Gutiérrez Trujillo

Renato Guzmao

Roberto Ham Chande

Mauricio Hernández Avila

Juan Eugenio Hernández Avila

Adolfo Hernández Garduño

Carlos Alfonso Hernández Girón

Ma. del Rocío Hernández Pozo

Bernardo Hernández Prado

Griselda Hernández Tepichín

Irva Hertz Picciotto

Martha Híjar Medina

Jane Hopping

José Guadalupe Huerta López

José Antonio Izazola Licea

Felicia Knaul

Gustavo Kourí Flores

Pablo Antonio Kuri Morales

Marina Lacasaña Navarro

Ana Langer Glass

Eduardo César Lazcano Ponce
Israel Lerman Garber

Susana Lerner Sigal

Malaquías López Cervantes

Olga López Rios

Francisco López Sosa

Rafael Lozano Ascencio

Ignacio Madrazo Navarro

Herlinda Madrigal Fritsch

Elsa Malvido Miranda

Homero Martínez Salgado

Carolina Martínez Salgado

Reynaldo Martorell

Rodolfo Méndez Vargas

Fernando Meneses González

Alejandro Mohar Betancourt

Raúl E. Molina Salazar

Miriam Muñoz de Chavez

Onofre Muñoz Hernández

Guillermina Natera Rey

Joel Navarrete Espinoza

Gustavo Nigenda López

Gustavo Olaiz Fernández

Patricia Olaya Contreras

Ursula Oswald Spring

Socorro Parra Cabrera

Carlos Paz Tres

Miguel Angel Peredo

Rafael Pérez Escamilla

Rogelio Pérez Padilla

Samuel Ponce de León Rosales

Carlos Posadas Romero

Julio Querol Vinagre

Velia A. Ramírez Amador

Teresita Ramírez Sánchez

Janine Madeleine Ramsey

Ricardo Alberto Rangel Guerra

Iván Restrepo Fernández

Sandra Reyes Frausto

Hortensia Reyes Morales

Vesta Richardson López-Collada

Camilo Ríos Castañeda

Octavio Rivero Serrano
Anthony Robbins

Mario Henry Rodríguez López

Jorge Luis Rosado

Irma Rosas Pérez

Steve Rothenberg

Dolores Saavedra Ontiveros

Eduardo Sada Díaz

Eduardo Salazar Martínez

Ana María Salinas Martínez

Jonathan M. Samet

Elsa Sarti Gutiérrez

Moisés Selman Lama

Teresa Shamah Levy

Tomas Schlenker

Juan José Luis Sienra Monge

Gonzalo Solís Cervantes

Fortino Solórzano Santos

Julio Sotelo Morales

José Luis Soto

Juan Alfredo Tamayo y Orozco

Roberto Tapia Conyer

Martha María Téllez-Rojo Solís

Luisa Elvira Torres Sánchez

Felipe Javier Uribe Salas

Patricia Uribe Zúñiga

Miguel Angel Vaca Marín

José Luis Valdespino Gómez.

Mario Humberto Vargas Becerra

Leopoldo Vega Franco

Leopoldo Vega Franco

Oscar J. Velázquez Monroy

Sofía Villa Contreras

Carlos A. Villanueva Díaz

Patricia Volkow Fernández

Liria Tatsuko Yamamoto Kimura

Lucía Bertha Yáñez Velasco

Alberto Ysunza Ogazón

Jorge Zavala Velázquez

Beatriz Zurita Garza

Víctor Zurita Saldaña 